\title{
BEHÖRDEN UND KÖRPERSCHAFTEN DER ARBEITS- UND SOZIALVERWALTUNG
}

Schriftgut aus der Arbeits- und Sozialverwaltung ist in staatlichen Archiven nur relativ wenig überliefert, weil viele Organe der Selbstverwaltung nicht zur Abgabe ausgesonderter Akten verpflichtet sind. Es ist daher zu erwarten, daß ihre Wirksamkeit in der Zeit von 1933 bis 1945 auch durch Bestände in Altregistraturen und Behördenarchiven bestehender Einrichtungen dokumentiert ist. Als ergänzende staatliche Überlieferung ist auf die Akten der Gewerbeaufsicht durch Behörden der allgemeinen inneren Verwaltung (Abschnitt 2.1.2.3) und der Arbeits- und Sozialgerichtsbarkeit (Abschnitt 3.2.5), ferner auf die Überlieferung der Deutschen Arbeitsfront (Abschnitt 9.3.1) zu verweisen.

\subsection{Reichsarbeitsministerium mit nachgeordneten Behörden und Einrichtungen}

\subsubsection{Reichsarbeitsministerium}

BA, Best. R 41

ZStA, Best. 39.01

Der Koblenzer Bestand enthält aus dem Ministeramt Franz Seldtes, der von 1933 bis Kriegsende das Ressort verwaltete, Brieftagebücher (6 Bde, 1933-1939) und Akten (29 Bde, 1933-1944) mit Eingaben vor allem in Personalangelegenheiten und von ehemaligen Mitgliedern des „Stahlhelm“ (16 Bde, 1933-1938), Aufzeichnungen über Sitzungen der Reichsregierung (1933-1936), des preuBischen Staatsministeriums (1933-1938), andere Ministerbesprechungen (1935-1943) und zwei Tagungen der Reichsarbeitskammer (1935/36), Reste allgemeiner Korrrespondenz (1938-1944) und von Schriftwechsel mit Ley und Reichsministerien (bis 1939), ferner einige Vorgänge über Zuständigkeitsfragen (1940-1944), die Reform der Altersversorgung (1940-1943) und Berichte des Generalbevollmächtigten für den Arbeitseinsatz.

Den Hauptteil des Bestandes in Koblenz bilden Akten der Hauptabteilung IV für Siedlungs- und Wohnungswesen, Städtebau und -planung. Ab 1940 wurde daraus in verschiedenen Etappen die Behörde des Reichskommissars für den sozialen Wohnungsbau Robert Ley, dessen Erlasse und Verordnungen in einer Sammlung überliefert sind (6 Bde, 1940-1944), während die Tätigkeit von Gottfried Feder als Reichskommissar für das Siedlungswesen (1934) und Staatssekretär im Reichswirtschaftsministerium nur durch wenige Akten dokumentiert ist (3 Bde, 1934-1935); nur das Sachgebiet Baupolizei und Stadtplanung blieb im Ministerium. Vorhanden sind Sammlungen von Erlassen (28 Bde, 1930-1942, überwiegend ab 1935), Unterlagen über Organisation und inneren Dienst (21 Bde, 1933-1944) und Sachakten (40 Bde betr. Wohnungsfürsorge, 1926-1936, auch im ZStA) vor allem aus folgenden Gebieten:

- Wohnungs- und Siedlungswesen (ca. 200 Bde) mit Eingaben einzelner Bauträger und Finanzierungsinstitute (17 Bde, 1933-1938), Unterlagen über Öffentlichkeitsarbeit (3 Bde, 1934-1937), Bewirtschaftung des Wohnungsfüsorgefonds und anderer Haushaltsmittel (ca. 30 Bde, 1933-1942), Kleinsiedlungen und Landarbeiterwohnungsbau (14 Bde, 1930-1941), Wohnsiedlungs- (27 Bde, 1932-1938) und Siedlungsplanungsgesetz 
(16 Bde, 1931-1939) sowie Bauanzeigen (1934-1942, mit Bebauungs- und Lageplänen) aus Berlin ( 11 Bde), den übrigen preuBischen Bezirken (47 Bde), dem Ruhrkohlenbezirk (8 Bde) und den anderen Ländern (32 Bde).

- Bau- und Bodenrecht (ca. 40 Bde, u. a. betr. Bodenreform und Enteignungen, meist 1933-1937) und seine Einführung wie auch sonstigen Reichsrechts im Saarland, eingegliederten Gebieten, dem Protektorat Böhmen-Mähren und dem Generalgouvernement (30 Bde).

- Bautechnik und Baustoffe, insbesondere Kosten- und Preisermittlung und -überwachung, Bewirtschaftung, Normung, Heizung (ca. 80 Bde, meist 1929-1937, dabei auch 3 Bde betr. Eingliederung der Architekten in die Reichskammer der bildenden Künste. 1934-1938).

- Errichtung von Wohnungen und anderen Bauten (1935-1942) für das Heer (30 Bde, u. a. auf Truppenübungsplätzen, für Munitionsanstalten und die Versuchsanstalt Peenemünde), Kriegsmarine (38 Bde, vor allem über Bauten in Wilhelmshaven und Kiel) und Luftwaffe (59 Bde, auch über Werke der Flugzeugindustrie).

- Reichs- und Landesplanung im allgemeinen (23 Bde), einzelnen Ländern und Regierungsbezirken (61 Bde, 1925-1938), im Siedlungsverband Ruhrkohlenbezirk (10 Bde, 1931-1937), Verkehrs- und Stadtplanung in Berlin und im übrigen Preußen (19 Bde, 1930-1937).

- Baupolizei (ca. 80 Bde), vor allem bei einzelnen militärischen Bauten (6 Bde, 1937 1942) und Lagern für Mineralöl (23 Bde, 1938-1942).

Geringeren Anteil haben Akten über Ausstellungen und Kongresse (16 Bde, bis 1937), Verkehrsplanung (4 Bde, 1933-1939), Industrieverlagerung (7 Bde, 1934-1939), Karten- und Luftbildwesen (7 Bde) sowie Luftschutz (17 Bde, 1933-1944).

Einen weiteren Schwerpunkt bilden in Koblenz Akten der Hauptabteilung $\mathbf{V}$ für Arbeitseinsatz, die ab 1942 - ebenso wie die Hauptabteilung III - dem beim Vierjahresplan ressortierenden Generalbevollmächtigten für den Arbeitseinsatz Fritz Sauckel (Reste privater Korrespondenz 1934-1945 im BA, Kl. Erw. 848) diente. Aus ihr sind vor allem Erlasse (24 Bde, 1939-1945), Unterlagen über Vorbereitungen für den Mob-Fall (11 Bde, 1936-1939), Wirtschaftslageberichte aus Berlin (4 Bde, 1937-1940) und besetzten Gebieten (5 Bde, 1941-1942), Handakten von Beamten (16 Bde, 1938-1945, u. a. betr. Einberufungen und Uk-Stellungen) und Akten über die Lenkung des Arbeitseinsatzes und die Sicherung des Kräftebedarfs überliefert. Sie betreffen die Einsätze von Arbeitskräften für Aufgaben besonderer staatspolitischer Bedeutung (10 Bde, 1939 1943), für die Rüstungsindustrie (21 Bde, 1936-1941), die Bauwirtschaft (28 Bde, 1937-1943), die Mineralöl- und Bunaproduktion (11 Bde, 1941-1942), den Bergbau (3 Bde, 1937-1941) und die Reichsbahn mit Zuliefererbetrieben (4 Bde, 1942), die politische Überprüfung von Beschäftigten in Rüstungsbetrieben (4 Bde, 1937-1940), die Einschränkung von Produktionen für den Zivilbedarf (4 Bde, 1941-1942) und nicht zuletzt den Einsatz von Frauen (5 Bde, 1939-1942), Kriegsgefangenen und Fremdarbeitern im allgemeinen (19 Bde, 1939-1944, mit Stimmungsberichten u. a. als Ergebnis der Postzensur durch die Auslandsbriefprüfstellen Köln, Hamburg, München, Wien und Berlin und die Zentralstelle für Telegramme Berlin 1942-1943) und die Anwerbung in den besetzten Ostgebieten (6 Bde, 1942-1943) und in Frankreich und dem übrigen Westeuropa (5 Bde, 1940-1944).

Von der Hauptabteilung II, Reichsversicherung und -versorgung, Wohlfahrtspflege, befinden sich in Koblenz nur Aktenreste (38 Bde, 1922-1944), jedoch in Potsdam eine relativ umfangreiche Überlieferung (ca. 1200 Bde nach 1933), vor allem 
über Kriegsbeschädigtenfürsorge im In- und Ausland (170 Bde, 1922-1943), Unterlagen zum Übergang der Zuständigkeit für die Versorgung von der Wehrmacht auf die Arbeitsverwaltung (345 Bde, 1929-1944) und über die Versorgung der Offiziere des Beurlaubtenstandes und der Mannschaften der alten Wehrmacht (143 Bde, 1926-1944). Von den dortigen Akten über die Sozialversicherung (ca. 1230 Bde, 1927-1943, mit Schwerpunkt bei der Invalidenversicherung) stammt etwa ein Viertel aus der Zeit nach 1933.

Die übrigen Hauptabteilungen sind demgegenüber in beiden Beständen nur mit wenigen Akten vertreten. In Potsdam gehören dazu eine relativ umfangreiche Überlieferung des Volkswirtschaftlichen Referates (ca. 50 Bde, 1930-1937, dabei 30 Bde mit Eingaben von Verbänden, Handelskammern und Einzelpersonen) und Akten mit folgenden Betreffen: Statistik, vor allem Lohnstatistik im Ausland (22 Bde, 1928-1937), Tarifund Arbeitsstreitigkeiten im Bergbau (20 Bde, 1922-1936); Betriebsräte: allgemeine Bestimmungen, Entscheidungen und Streitfälle, Schutz- und Strafbestimmungen (39 Bde, 1920-1936); Erwerbslosenangelegenheiten, Arbeitsnachweise (6 Bde, 1927-1939), Gewerbewesen (35 Bde, 1925-1936), Presse, allgemeiner Schriftverkehr (12 Bde, 1932 1936), Schlichtungswesen (58 Bde, 1920-1936), Sozialpolitik (12 Bde, 1922-1936), Vereinswesen (34 Bde, 1926-1936).

Der Rest des Koblenzer Bestandes enthält aus der Zuständigkeit der Hauptabteilung I für allgemeine Angelegenheiten und Dienststellenverwaltung Runderlasse (3 Bde, 1939-1944), Unterlagen über inneren Dienst (7 Bde, 1929-1945), Haushalt (7 Bde, 1930 1945), Personalangelegenheiten (ca. 55 Bde, u. a. über das Reichslager für Beamte Bad Tölz 1938-1941, Berufsbeamtengesetz 1933-1943, Verleihung von Kriegsverdienstkreuzen und anderen Orden bis 1943), Personalakten (u. a. von Präsidenten der Landesarbeitsämter und Reichstreuhändern der Arbeit, weitere Personalakten im ZSLA).

Aus der Hauptabteilung III, Arbeitsrecht, Arbeitsschutz, Gewerbeaufsicht stammen in Koblenz noch Erlasse (1933-1942), Unterlagen über personelle Besetzung von Ehrengerichten (1935-1943), Handakten (21 Bde, 1926-1942) des Staatssekretärs Johannes Krohn über Organisationsangelegenheiten, ArbeitsbeschaffungsmaBnahmen, Verhältmis zur DAF und sozialpolitische Pläne Leys, anderer Beamter (11 Bde) über Lohnpolitik (1937-1945).

Als Ergänzungsüberlieferung zu nennen sind die Akten des Verfahrens gegen Sauckel im Nürnberger ProzeB gegen die Hauptkriegsverbrecher, zahlreiche einzeln veröffentlichte Vorschriften für Arbeitseinsatz, Führung von Arbeitsbüchern, Beschäftigung von Ausländern und Jugendlichen, das Handbuch des Generalbevollmächtigten für den Arbeitseinsatz (Bd. 1, 1944), statistische Übersichten (z. B. über die Ergebnisse der Arbeitsbucherhebungen 1940 und 1941), die „Handbücherei des Wohnungs- und Siedlungswesens", die Schriftenreihe „Arbeit und Gesundheit" und das „Reichsarbeitsblatt" (bis 1941 Amtsblatt des Reichsarbeitsministeriums, des Reichsversicherungsamtes und der Reichsversicherungsanstalt für Angestellte) mit Teilen für amtliche Mitteilungen, Reichsversicherung, Arbeitsschutz, Bekanntmachungen über Tarifordnungen und Richtlinien für Betriebsordnungen und Einzelarbeitsverträge und dem Reichsversorgungsblatt, ferner das „Nachrichtenblatt des Reichsarbeitsministeriums“ (sämtlich - unvollständig - im BA, Best. RD 89).

Lit:: SCHRIFTEN ZUM STAATSAUFBAU 46. 1940.- A. KRANIG: Lockung und Zwang. 1983. - M.-L. RECKER: Nationalsozialistische Sozialpolitik im Zweiten Weltkrieg. 1985. - Dies.: Der Reichskommissar für den sozialen Wohnungsbau. 1986. - U. HERBERT: Fremdarbeiter. 1985. - P. W. BECKER: Fritz Sauckel 
- Generalbevollmächtigter für den Arbeitseinsatz. 1989. - D. EICHHOLTZ: Die Vorgeschichte des Generalbevollmächtigten für den Arbeitseinsatz. 1973. - T. MASON: Arbeiterklasse und Volksgemeinschaft. 1975. - D. PETZINA: Die Mobilisierung deutscher Arbeitskräfte vor und während des Zweiten Weitkriegs. 1970. - W. NAASNER: Neue Machuentren in der deutschen Kriegswirschah 1942-1945. 1989.

\subsubsection{Reichskommissar für Arbeitsbeschaffung ZSLA, Best. 39.05}

Die Aufgaben des Anfang Dezember 1932 berufenen und bereits im März 1933 entlassenen Reichskommissars Günter Gereke, zusätzliche Arbeitsplätze durch Notstandsarbeiten und freiwilligen Arbeitsdienst zu schaffen und den Bau von Wohnungen, vorstädtischen Klein- und landwirtschaftlichen Siedlungen zu fördern, gingen im Juli 1933 auf das Reichsarbeitsministerium über. Die bis dahin entstandenen Akten des Bestandes betreffen:

Allgemeine Verwaltungsangelegenheiten einschl. Presse (29 Bde), Haushalt (11 Bde), Arbeitsbeschaffungsprogramm (46 Bde, bis 1934), Finanzierung der Arbeitsbeschaffung einschl. Darlehensgesuche und Anträge der Länder ( 93 Bde, bis 1937), vorstädtische Kleinsiedlungen (18 Bde), ländliche Siedlungen (8 Bde), Wohnungsbau (22 Bde), freiwilligen Arbeitsdienst (4 Bde).

Lit.: G. GEREKE: Ich war königlicher preuBischer Landrat. 1970.

\subsubsection{Nachgeordnete Behörden und Einrichtungen}

Dem Reichsarbeitsminister unterstanden unmittelbar oder als Dienstaufsichtsbehörde zahlreiche Dienststellen und Selbstverwaltungskörperschaften mit zentraler und regionaler Zuständigkeit, wobei in den Ländern gewisse, früher z. T. Arbeitsministerien übertragene Kompetenzen von den Wirtschaftsministerien, z. B. in Bayern. wahrgenommen wurden. Außer von den in den folgenden Abschnitten noch genannten Behörden fehlt auch jede Überlieferung von der Reichsstelle für Arbeitsschutz, dem Reichsausschuß der Kriegsbeschädigten- und Kriegshinterbliebenenfürsorge, der Zentralaufsichtsstelle für Sprengstoff- und Munitionsfabriken, Deutschen Akademie für Bauforschung mit Franz-Seldte-Institut Magdeburg, der Reichsstelle für Baustatik und weiteren sechs Einrichtungen nach dem Stand von 1942 (für die Gerichte vgl. Abschnitte 3.2.5 und 3.2.6.6).

\subsubsection{Deutsche Akademie für Wohnungswesen}

ZStA. Best. 40.02

Die Akademie wurde im November 1941 unter Einbeziehung ähnlicher Einrichtungen wie der Deutschen Gesellschaft für Wohnungswesen e.V. und der Reichsstelle für Wohnungswesen gegründet, um wissenschaftliche und praktische Untersuchungen durchzuführen, für den Wohnungsbau zu werben, Typisierung, Normung und andere RationalisierungsmaBnahmen beim Bauen zu fördern. Der Bestand (ca. 260 Bde) enthält auch einige Akten des Reichswohnungskommissars (6 Bde), einzelne zur Geschäftsführung (14 Bde) und zur Wirtschaftlichkeit des Bauwesens (18 Bde), vornehmlich jedoch Unterlagen zur Siedlungsgestaltung, Städteplanung und Gestaltung der Wohngebiete (42 Bde, 1915-1928, 1930-1947), zur Konstruktion und Normung im Hausund Wohnungsbau (142 Bde, 1914-1948) sowie zu einzelnen Baustoffen (37 Bde, 1922 1947). 


\subsubsection{Arbeitsverwaltung}

\subsection{Reichsanstalt für Arbeitsvermittlung und Arbeitslosenversiche- rung ZSLA, Best. 39.03 \\ BA, Best. R 163}

Die 1927 errichtete Reichsanstalt wurde Anfang 1939 als Abteilung V in das Reichsarbeitsministerium eingegliedert, ihr Präsident Syrup (Unterlagen für seine Publikation über die Geschichte der Sozialversicherung mit unveröffentlichten Teilen im BA, Kl. Erw. 636) zum Staatssekretär ernannt. Die vorher und nachher geführten Akten befinden sich, soweit sie den Krieg überstanden, im ZSLA. Sie haben folgende Schwerpunkte:

Arbeitslosenversicherungsbeiträge, Krankenkassen (61 Bde, 1927-1938), Arbeitslosenversicherung im Bergbau (2 Bde, 1931-1937), HilfsmaBnahmen für Städte (11 Bde, 1925-1938), Dienstbetrieb (3 Bde, 1932-1937), Arbeitsbeschaffungsprogramm (101 Bde, 1928-1938), Landarbeiterwohnungsbau (8 Bde, 1930-1937), Akademiker- und Künstlerhilfe (14 Bde, 1931-1937), Haushalt und Finanzierung (18 Bde, 1928-1937), Neues Arbeitsbeschaffungsprogramm, Freiwilliger Arbeitsdienst (28 Bde, 1932-1937), Durchführung von Notstandsarbeiten (95 Bde, 1933-1940), Drucksachen und Rundschreiben (22 Bde, 1929-1941), Personalakten.

Der Koblenzer Bestand beschränkt sich für die NS-Zeit auf Handakten über Grundsatzfragen der Personalverwaltung (50 Bde, 1928-1946) und aus Empfängerüberlieferung zusammengestellte Serien der Rundschreiben des Präsidenten (6 Bde, 1930-1938) und der „Dienstlichen Mitteilungen“ (8 Bde, 1933-1937). Er wird ergänzt (Best. RD 89) durch das „Dienstblatt“ (fortgesetzt als „Dienstblatt des Reichsarbeitsministeriums für die Landesarbeitsämter und Arbeitsämter“, dann als „Runderlasse für den Arbeitseinsatz, die Reichstreuhänder der Arbeit und die Gewerbeaufsichtsverwaltung“, 8 Bde, 1938-1945), durch „Jahresberichte“ (1. 4. 1933-31. 3. 1936) und sechs (unvollständige) Jahrgänge von „Der Arbeitseinsatz im Deutschen Reich“ (1938-1942, 1944). Von der außerdem bestehenden Reichsstelle für Arbeitsvermittlung ist nichts überliefert.

Lit: F. SYRUP: Hunder Jahre staatiche Sozialpolitik. 1957. - H. KAHRS: Die ordnende Hand der Arbeitsämter. 1990.

\subsection{Reichstreuhänder der Arbeit}

Aus der Tätigkeit der bereits 1933 für (1942) 23 Wirtschaftsgebiete (davon 9 im späteren Gebiet der Bundesrepublik und Berlin) eingesetzten Reichstreuhänder, deren Funktionen ab September 1943 den Präsidenten der neu errichteten Gauarbeitsämter übertragen wurden, sowie der Sondertreuhänder für den öffentlichen Dienst und die Heimarbeit bei der Festsetzung von Löhnen und Arbeitsbedingungen ist kaum Schriftgut erhalten geblieben. Sie wird vor allem durch die im „Reichsarbeitsblatt“ veröffentlichten Bekanntmachungen über Tarifordnungen und Richtlinien für Betriebsordnungen und Einzelarbeitsverträgen und folgende Reste von Akten und regionalen, zweimal monatlich erscheinenden Mitteilungsblättern dokumentiert:

Reichstreuhänder für den öffentlichen Dienst „Amtliche Mitteilungen“" (BA, RD 93, Jahrgang 1, 1939 - 7, 1945).

Reichstreuhänder der Arbeit Bayern, München „Amtliche Mitteilungen“ (BA, RD 93, unvollständige Jahrgänge 1, 2, 4-9, 19351936, 1938-1943, auch als gemeinsame Veröffentlichung der Präsidenten der Gau- 
arbeitsämter und Reichstreuhänder für Franken, Mainfranken, Oberbayern und Schwaben).

\section{Reichstreuhänder der Arbeit Hessen, Frankfurt}

Akten (1934-1944) über Löhne und Gehälter bei 16 einzelnen Firmen, vorwiegend Banken und Versicherungen, statistische Unterlagen aus dem Bezirk der Handwerkskammer Wiesbaden (WI, in Abt. 483).

\section{Reichstreuhänder der Arbeit Niedersachsen, Hannover}

In Schriftgut des 1943 errichteten Gauarbeitsamtes Weser-Ems, seit 1945 Landesarbeitsamtes Bremen (HB, Best. 4,95) befindet sich eine umfangreiche Sammlung von Unterlagen (1934-1945), die aus der Zuständigkeit des Reichstreuhänders stammen. Sie betreffen Löhne, Arbeitszeit- und Urlaubsregelungen in der Land- und Forstwirtschaft, der Fischerei, der Industrie der Steine und Erden, der Chemischen, Metall-, Textil- und Papierindustrie, des Vervielfältigungs-, Nahrungs- und Genußmittel-, Bekleidungs-, Bauund Bauneben- und des Reinigungsgewerbes, des Groß- und Einzelhandels, des Bankund Versicherungs-, Verkehrs- und Gaststättengewerbes. Speziell sind Anordnungen für die Entlohnung bei der Organisation Todt und den NSKK-Transporteinheiten Todt/Speer (auch von „Ostlegionären“) und für Musiker, Artisten und Schauspieler (u. a. bei der Truppenbetreuung) vorhanden, ferner ,Amtliche Mitteilungen“ (HB, Amtsdrucksachen, Jahrgang 2, 1936, ab Nr. 14-7, 1941).

Reichstreuhänder der Arbeit Nordmark, Hamburg $\mathrm{HH}$, Best. 356-4 Akten über 15 einzelne Unternehmen (Banken, Versicherungen, Handelsbetriebe. Gaststätten, Industriebetriebe, 1934-1947) und über lohnordnende Maßnahmen während des Krieges bei 11 weiteren Firmen der Industrie (1942-1945) sowie (auch über Leistungssteigerungen und Arbeitsbedingungen) bei Seeschiffswerften im allgemeinen und einzelnen (11 Bde, 1941-1945), ferner Aktenreste über Arbeitsrecht, Eisen- und Metallindustrie, Schiffahrt und Hafenarbeiter, „Amtliche Mitteilungen“ (Jahrgang 2, 1935, in Best. 614-2, Jahrgänge 5-8, 1938-1941, unvollständig BA, RD 93).

Reichstreuhänder der Arbeit Südwestdeutschland, Karlsruhe Tarifakten für einzelne Wirtschaftszweige und Berufe, u. a. Textilindustrie, Lebensmittelbetriebe (1935-1945) und Einzelfälle von Löhnen und Gehältern (1944) sowie Monatsberichte (Dez. 1942-Jan. 1945) nur für Baden (FR), .Amtliche Mitteilungen“" (BA, RD 93, unvollständige Jahrgänge 1-3. 1935-1937, für Baden 18 Nrn von Jahrgang 2, 1944).

\section{Reichstreuhänder der Arbeit Westfalen-Niederrhein, Essen}

Die bei der Preisbildungsstelle für Schaumburg-Lippe über einzelne Branchen geführten Akten (ca. 180 Bde, vgl. oben Abschnitt 6.1.2.2) enthalten jeweils am Anfang „Anordnungen und Schriftverkehr" des Reichstreuhänders der Arbeit in Essen (1936-1944, überwiegend 1938-1944), ,Amtliche Mitteilungen“ (Jahrgänge 1-10, 1934-1943 Juli) in der Bibliothek des Westfälischen Wirtschaftsarchivs Dortmund.

\section{Reichstreuhänder der Arbeit Westmark, Saarbrücken} „Amtliche Mitteilungen“ (BA, RD 93, Jahrgang 9, 1943).

Von folgenden Präsidenten von Gauarbeitsämtern als Reichstreuhändern sind einzelne Nummern von „Amtlichen Mitteilungen“ vorhanden (BA, RD 93): 
Bayreuth (2, 1943, 1945), Berlin (30, 1943-1944), Düsseldorf (Jahrgang 1, 1944), Hamburg (24, 1944-1945), Rhein-Main und Kurhessen (nur Beiheft: Die Betriebsverlagerung. Stand Oktober 1944), Südhannover-Braunschweig (1, 1944), Köln-Aachen (11, 1944, außerdem einzelne Rundschreiben 1943/44 D, in Best. RW 23 und G 64), Schleswig-Holstein (Jahrgang 1, 1943).

\subsection{Landesarbeitsämter}

Von den 13 Landesarbeitsämtern, die 1942 für Berlin und Wirtschaftsgebiete im späteren Gebiet der Bundesrepublik zuständig waren, und den danach jeweils für einen Gau errichteten Gauarbeitsämtern sind ebenfalls nur wenige Akten in die staatlichen Archive gelangt. Sie verteilen sich auf folgende Provenienzen:

\section{Landesarbeitsamt (Nord- und Süd-)Bayern, Nürnberg und München}

Arbeitsmarkt- und Lageberichte (1933-1936) sowie Schriftgut der Werbekommission in der Ukraine sind in Akten des Arbeitsamtes Freising (MSt) überliefert, der Pressedienst (1933-1935) bei Schriftgut der DAF (BAM), außerdem für Nordbayern die Jahresarbeitseinsatzstatistik 1941 und „Mitteilungen“ (1941-1943, N, Rep. 251 I).

Landesarbeitsamt Niedersachsen, Hannover

H, Best. Hann. 275

Der Bestand enthält Unterlagen über Organisation und Verwaltung (9 Bde, darin Anweisungen 1932-1934, Aufzeichnungen über Dienstbesprechungen der Direktoren ab 1936, Lageberichte an den Reichsarbeitsminister ab 1939), Statistik (9 Bde, 1933-1958, u. a. über Ergebnisse der Berufszählung 1933 und über Wirtschaftslage in den Bezirken Hannover und Hildesheim ab 1934), über den öffentlichen Dienst (32 Bde. 19361948) und 28 einzelne Gewerbezweige (1922-1948, dabei über Arbeitsbedingungen in der Lufffahrtindustrie ab 1940), weitere Sachakten (ca. 70 Bde) u. a. betr. Tarife und Schlichtungen (ab 1936 bzw. 1938 mit Berichten der Lohnüberwachungsstellen ab 1943), Maßnahmen zur Erhaltung der Arbeitsdisziplin (ab 1938) und Leistungssteigerung (1941-1945), Unterbringung von Kriegsteilnehmern (ab 1939), Arbeitsbedingungen für Juden, Polen und Zigeuner (1938-1944).

\section{Landesarbeitsamt Rheinland, Köln}

Die Überlieferung beschränkt sich auf wenige Erlasse und Berichte über Arbeitseinsatzlenkung und -lage (D, in Best. RW 23, RW 86, 4 Bde, 1939-1944) und Vorakten des Landesarbeitsamtes Nordmein-Westfalen $(\mathrm{D}-\mathrm{K})$ über die Prüfung der Arbeitsämter Solingen, Velbert und Wuppertal (1939).

\section{Landesarbeitsamt Schleswig-Holstein, Kiel}

SL, in Abt. 580

In einer vor 1933 beginnenden und nach 1945 fortgesetzten Überlieferung befinden sich einzelne Akten über Arbeitsämter (ab 1933), Arbeitslosenversicherung und -hilfe (ab 1938), Zusammenarbeit mit den Sozialversicherungsträgern und Fürsorgeverbänden (1939-1947), Lohnausfallvergütung wegen Fliegeralarm (ab 1938), Verlagerung von Industriebetrieben (1944-1955).

\section{Landesarbeitsamt Westfalen, Dortmund}

Vorhanden sind (MS, 10 Bde) Erlasse des Reichsarbeitsministers (1933-1944), des Beauftragten für den Vierjahresplan (1938-1944), des Reichsverteidigungskommissars Westfalen-Süd (1943-1944) und anderer Reichsbehörden und Akten (D - K, in Akten 
des Landesarbeitsamtes Nordrhein-Westfalen) über die Prüfung der Arbeitsämter Bottrop (1939), Detmold (1939-1946), Recklinghausen (1937, 1942) und Rheine (1939).

Landesarbeitsamt Württemberg, Stuttgart

LB, Best. K $310 / 1$

Aus der Zeit vor 1945 enthält der Bestand nur einzelne Rundschreiben und Unterlagen über Personalangelegenheiten und wertschaffende Arbeitslosenhilfe (1928-1941).

\subsection{Arbeitsämter}

Nur von folgenden, in der Regel für mehr als einen Stadt- oder Landkreis zuständigen Arbeitsämtern (z. B. bestanden für 62 Stadt- und Landkreise der Rheinprovinz 39 Arbeitsämter) ist Schriftgut aus der NS-Zeit überliefert (zu darin enthaltenen Arbeitsdienstakten vgl. Abschnitt 7.2.2):

Arbeitsamt Ahlen (Westfalen)

MS

Statistik der Arbeiter und Angestellten (1943-1944).

Arbeitsamt Ahrweiler

KO, Best. 570/1

Statistik (10 Bde, 1934), Arbeitsvermittlung (4 Bde, ab 1944), Kindergeldzahlungen (6 Bde, ab 1932), Berufsnachwuchs (2 Bde, ab 1940).

Arbeitsamt Berlin-Mitte

B, Rep. 242

Planung und Durchführung der Lenkung des Arbeitseinsatzes im Krieg, Uk-Stellungen und Dienstverpflichtungen, personelle Mobilmachung der NSDAP (61 Bde, ab 1935, mit Listen von Rüstungsbetrieben).

\section{Arbeitsamt Bielefeld}

DT, Best. D 52

Einzelne Vorakten u. a. über Berufsberatung, psychologische Untersuchungen, Prüfung der Bedürftigkeit (ab 1937).

Arbeitsamt Bruchsal

KA, Abt. 460

Berichte über Arbeitsmarkt und Arbeitseinsatz (1933-1939) und Akten (insgesamt 91 Bde) u. a. betr. Arbeitsvermittlung (1937-1939), Zusammenarbeit mit der NSDAP und dem Reichstreuhänder der Arbeit (1934-1938), Einsatz von Strafgefangenen (1934), Wohnungsbauprogramm (1930-1937), politische Überprüfung von Arbeitsuchenden (1936-1939), Bekämpfung der Landflucht (1934-1939), Wanderarbeiter und ausländische Landarbeiter (1932-1939), Abwanderung ins Ausland (1933-1939).

Arbeitsamt Deggendorf

LA

Erhebungen über FAD-Einsatz (1933-1934) und Wohlfahrtsempfänger (1936-1938), Industrieberichterstattung (1942-1944).

Arbeitsamt Detmold

DT, Best. D 52

Jahresstatistiken (12 Bde, 1935-1949).

Arbeitsamt Dortmund

MS

Dienststellenverwaltung und allgemeine Anordnungen (16 Bde, 1932-1945), Personalangelegenheiten (15 Bde, 1933-1945), Festsetzung von Tarifen und Löhnen (6 Bde, 1933-1944). 
Arbeitsamt Düsseldorf

ISD

Arbeitskarten über 1059 französische Fremdarbeiter (1940-1945).

Arbeitsamt Freising

MSt

Anwerbung und Einsatz von Ostarbeitern, insbes. aus dem Gebiet Tschernigow im Generalbezirk Nikolajew (Ukraine) durch den Arbeitseinsatzstab Graf Spreti (17 Bde, 1941-1943).

Arbeitsamt Gelsenkirchen

MS

Dienststellenverwaltung, allgemeine Anordnungen, Direktorenbesprechungen ( 8 Bde. 1933-1945).

Arbeitsamt Herford

DT, Best. D 52

Einzelne Vorakten über Arbeitsbeschaffung, u. a. durch Autobahnbau, Vermittlung entlassener Strafgefangener, Industrie der Steine und Erden (ab 1931).

Arbeitsamt Idar-Oberstein

KO, Best. 570/8

Dienststellenverwaltung (23 Bde, ab 1933, dabei Unterlagen über Direktorenkonferenzen), Einsatz des RAD (1942), Heimarbeit (2 Bde, 1935-1944), Einsatz von Juden (1941-1944) und Fremdarbeitern (17 Bde, 1941-1945).

Arbeitsamt Iserlohn

MS

Arbeitsmarkt- (3 Bde, 1933-1936) und Wochenberichte an die Gauleitung Westfalen-Süd (1934-1935).

Arbeitsamt Kempten

A

Organisations- und Personalangelegenheiten, Haushalts- und Rechnungswesen (25 Bde, 1933-1945).

Arbeitsamt Lippstadt

MS

Monatsberichte (1935).

Arbeitsamt Lörrach

FR

Dienststellenverwaltung (6 Bde, vor 1939).

Arbeitsamt Ludwigshafen

SP, Best. O 4

Dienststellenverwaltung (ca. 200 Bde, 1929-1945), u. a. Anordnungen der Reichsanstalt, Sitzungsprotokolle, Prüfung durch Landesarbeitsamt, Errichtung von Durchgangslagern für Ausländer, Personalangelegenheiten (auch Prüfungen, Lehrgänge an der Reichsschule Potsdam-Babelsberg).

Arbeitsamt Lünen

Notstandsarbeiten (6 Bde, 1935-1937).

Arbeitsamt Mayen

KO, Best. 570/5

Unterlagen über den Arbeitsmarkt in einzelnen Wirtschaftszweigen (21 Bde ab 1941).

Arbeitsamt Memmingen

Aktenreste über Wohlfahrtsaufwendungen der Gemeinden, Zusammenarbeit mit Reichsnährstand, ausländische Arbeiter (1933-1936). 
Arbeitsamt Neumünster

SL, Abt. 581

Dienststellen- und Aufgabenverwaltung (30 Bde, 1932-1963).

Arbeitsamt Neunkirchen (Saar)

Statistiken und Strukturdaten zur Zahl der Beschäftigten nach Wirtschaftszweigen und Vorbildung (17 Bde, 1935-1944).

Arbeitsamt Paderborn

DT, Best. D 52

Durchführung von Einzelprojekten der wertschaffenden Arbeitslosenhilfe (ca. 30 Bde, 1933-1941).

Arbeitsamt Rosenheim

MSt

Einzelne Vor- und Nachakten, u. a. Berufsberatung, Landhilfe, Fortbildung von Arbeitslosen (1927-1944), Arbeitsmarktberichte (1933-1952).

Arbeitsamt Schwandorf

$\mathrm{AM}$

Organisation und Dienststellenverwaltung (5 Bde, 1932-1937), Unterbringung „Alter Kämpfer" der NSDAP (4 Bde, 1933-1936).

Arbeitsamt Soest

Allgemeine Anordnungen (1929-1941), Sitzungs- (2 Bde, 1929-1945) und Schulungsunterlagen (2 Bde, 1935-1945), Monatsberichte (1936).

\section{Arbeitsamt Stade}

STD, Rep. 260

Vorakten (ab 1928) u. a. über Organisation und Dienststellenverwaltung (32 Bde). Arbeitsbeschaffungsmaßnahmen (15 Bde, u. a. zum Bau der Reichsautobahnen), Berufsberatung und -bildung (5 Bde), Arbeitslosenversicherung (17 Bde).

Arbeitsamt Straubing

Statistiken über Arbeitslose, Bauwirtschaft, Heimarbeit, Kurzarbeit, Landhilfe. Vermittlung und Berufsberatung (8 Bde, 1933-1945).

Arbeitsamt Watenstedt-Salzgitter

Arbeitskarten (auch von anderen Arbeitsämtern in Niedersachsen) über 5612 Fremdarbeiter.

Arbeitsamt Wuppertal

$\mathrm{D}-\mathrm{K}$

Aktenrest (6 Bde, 1941-1951) aus Organisations- und Verwaltungsangelegenheiten (Durchgangslager für Zivilpersonen), Arbeitsvermittlung (Frauen, Asoziale, Kulturberufe).

\subsubsection{Versicherungsverwaltung}

Träger der gesetzlichen Sozialversicherung waren auch unter dem NS-Regime vornehmlich für die Altersversicherung die Reichsversicherungsanstalt für Angestellte, die Landesversicherungsanstalten in den preußischen Provinzen und den Ländern für die Invalidenversicherung der Arbeiter und die Reichsknappschaft für die Arbeitnehmer des Bergbaus, für die Unfallversicherung die Berufsgenossenschaften, für die Krankenversicherung die Allgemeinen Orts- und Landkrankenkassen und die privaten Ersatzkassen für Angestellte. Sie verwalten ihr Schriftgut selbst, ebenso die Kassenärztlichen und 
Kassenzahnärztlichen Vereinigungen. Die folgenden Bestände in staatlichen Archiven stammen aus den für die Aufsicht zuständigen Behörden, aus vom Staat für seine Bediensteten geschaffenen Versicherungseinrichtungen oder wurden bei der Liquidierung aufgelöster Verbände erworben.

\subsubsection{Reichsversicherungsamt}

BA, Best. R 89

In der sehr umfangreichen, mit der Gründung des Amtes 1884 einsetzenden Überlieferung sind die zwischen 1933 und 1945 geführten Akten nur schwer auszugrenzen. Sie sind aus allen Organisationseinheiten der Büroabteilungen in folgender Gliederung vorhanden (zu den Akten aus der Rechtsprechung des Amtes vgl. Abschnit 3.2.5.2.1): Zentralbüro: Organisation des Amtes und der Abteilungen, Haushalts-, Kassen- und Rechnungswesen (u. a. Vergabe von Darlehen aus der Reichsarbeitskasse), Publikationen, Sozialgesetzgebung und Wohlfahrtspflege, Verkehr mit anderen Behörden und Organisationen, Konferenzen, Kongresse, Ausstellungen, Personalangelegenheiten (dabei 4 Bde betr. Berufsbeamtengesetz 1933-1942, Einstellung „Alter Kämpfer“ 1933-1943). Unfallversicherungsabteilung (ca. 5000 Bde, 1884-1944): Grundsätzliche Vorschriften der Reichsversicherungsordnung (u. a. Gesetzgebung, Verwaltung und Geschäftsführung der Oberversicherungsämter), gewerbliche, landwirtschaftliche und See-Unfallversicherung, Aufsicht über die Berufsgenossenschaften (mit Satzungen und Geschäftsberichten), ihre Haftpflichtversicherungsanstalten und Verbände, Unfallverhütung.

Kranken- (325 Bde, 1912-1944, u. a. Organisation der Krankenkassen, Zulassung von Ersatzkassen) und Invalidenversicherungsabteilung (2400 Bde, 1890-1944, vor allem Aufsicht über die Landesversicherungsanstalten, mit Satzungen und Geschäftsberichten, auch über Seekasse und Versicherungsanstalt der Reichsbahn, Maßnahmen zur Gesundheitsfürsorge).

Knappschafts- und (in geringerem Umfang) Angestelltenversicherungsabteilung (mit Akten über Ersatzkassen und wenigen Akten über Arbeitslosenversicherung).

Lit.: FINDBÜCHER zu Beständen des Bundesarchivs. Bd. 32. 1987. 1988.

\subsubsection{Reichsverband Deutscher Rentenversicherungsträger}

BA, Best. R 40

Der Bestand (572 Bde, 1919-1945) umfaBt neben Protokollen der Verbandstage und des Ständigen Ausschusses Akten über die gesetzlichen Grundlagen der Sozialversicherung, über Verfassung und Verwaltung der Landesversicherungsanstalten, der Reichsbahnversicherungsanstalt, der Seekasse und der Reichsknappschafi, das Renten- und Beitragsverfahren, Gesundheitsfürsorge in ihren Heilstätten und Kureinrichtungen, ausländische Sozialversicherung und andere zwischenstaatliche Beziehungen.

Lit.: FÜNFUNDSIEBZIG Jahre Landesversicherungsanstalten. 1966.

\subsubsection{Reichsversicherungsanstalt für Angestellte $\quad$ BA, Best. R 112}

Der Hauptteil ihrer Überlieferung befindet sich bei der Bundesanstalt für Angestelltenversicherung. Der Bestand enthält einerseits bis 1934 reichende Akten aus dem Zentralbüro der Präsidialabteilung über Aufgaben, Organisation und Tätigkeit von Verwaltungsrat und Direktorium (277 Bde, ab 1912), andererseits über die Aufsicht über 19 Ersatzkassen der Krankenversicherung für Angestellte (85 Bde, 1940-1944).

LiL: FÜNFZIG Jahre Angestelltenversicherung. 1962. 


\subsubsection{Reichsknappschaft}

BA, Best. R 156

Der Bestand enthält Verträge und Rundschreiben (46 Bde, 1933-1936) und durchgehend von 1923 bis 1945 gefuihrte Akten aus den Sachgebieten Gesetzgebung und Satzung (360 Bde), Verwaltung (404 Bde), Versicherung (404 Bde), Gesundheitsfürsorge (208 Bde), Kassen- und Rechnungswesen (373 Bde), zwischenstaatliche Beziehungen (201 Bde).

\subsubsection{Reichsbahnversicherungsanstalt}

ZStA, Best. 43.09

Der Bestand (ca.1,5 lfm) ist noch nicht geordnet und verzeichnet.

\subsubsection{Reichsverband der Ortskrankenkassen $\quad$ BA, Best. R 42 I}

Die wenigen Restakten betreffen Organisation und Dienststellenverwaltung (14 Bde, 1933-1945, mit Rundschreiben und Mitteilungen), Sozial- und Krankenversicherung im allgemeinen (14 Bde, 1942-1945, dabei auch Verwaltungsvereinfachung, Versicherung von Fremdarbeitern), Personalangelegenheiten der Landesgeschäftsstellen (18 Bde, 1943-1945), Abwicklung der Krankenkassenverbände der Rheinprovinz (13 Bde, 1933 1943) und die Bewertung von ca. 30 einzelnen Kur- und Genesungsheimen (1937-1940).

LiL: S. LEIBFRIED, F. TENNSTEDT: Benufsverbote und Sozialpolitik 1933. 1979.

\subsubsection{Betriebskrankenkasse des Reichs BA, Best. R 42 II}

Die Akten der erst 1939 durch Vereinigung mehrerer Kassen für versicherungspflichtige Arbeitnehmer von Reichsbehörden, Wehrmacht, Polizei, RAD, Organisation Todt und Technischer Nothilfe errichteten Kasse stammen von den Zweigstellen Köln, Koblenz und den Haag und enthalten neben Erlassen und Rundschreiben ( 7 Bde) vor a!lem Unterlagen über Organisation und Dienststellenverwaltung (47 Bde) und Geschäftsund Rechnungsführung (15 Bde).

\subsubsection{Reichsausführungsbehörde für Unfallversicherung}

BA, Best. R 152

Der Bestand enthält (mit Vorakten aus der Zeit vor Errichtung der Behörde 1940) Unterlagen zur Organisation (10 Bde, mit Rundschreiben), Unfallversicherung für Bedienstete des Reichs und Unfallvertütung im allgemeinen ( 9 Bde), bei einzeinen Behörden (26 Bde, u. a. Reichsministerien, Reichsrundfunkgesellschaft, Luftschutz), in eingegliederten und besetzten Gebieten (18 Bde) und in Sonderfallen ( 7 Bde, dabei Kriegsgefangene, Beschäftigte bei Schanzarbeiten und beim Behelfsheimbau), ferner über 14 Einzelfälle.

\subsubsection{Oberversicherungsämter}

Die meisten der zur Aufsicht über die einzelnen Träger der Sozialversicherung seit 1911 bestehenden Oberversicherungsämter waren formal in die Behörden der preuBischen Regierungspräsidenten eingegliedert; soweit sie wie die heutigen Sozialgerichte in Prozessen über Versicherungsansprüche entschieden, ist die Überlieferung darüber in Abschnitt 3.5.2.2.4 behandelt. Aus der Verwaltungstätigkeit gibt es folgende Bestände und Teilbestände:

Oberversicherungsamt Aurich

AUR, in Rep. 16 Akten über Organisation und Dienststellenverwaltung (7 Bde, 1912-1951) und über Betriebs-, Orts- und Landkrankenkassen sowie Begräbniskassen (55 Bde, 1900-1955). 
Oberversicherungsamt Berlin

B, Rep. 58

Generalakten (16 Bde, davon 7 Bde mit Geschäftsübersichten, 1933-1945).

Knappschafts-Oberversicherungsamt Clausthal $\quad H$, Best. Hann. 121 Generalakten (81 Bde, 1886-1956), dabei über Besprechungen der Direktoren der Knappschafts-Oberversicherungsämter (1925-1936).

Oberversicherungsamt Detmold

DT, Best. L 80 I b A

Generalakten (ca. $100 \mathrm{Bde}$ ) über Dienststellenverwaltung, Berufsgenossenschaften, einzelne Orts-, Land- und Betriebskrankenkassen, Invalidenversicherung.

Oberversicherungsamt Lüneburg

$\mathrm{H}$, in Best. Hann. 180 Lüneburg Akten über das Berufsbeamtengesetz (1933-1937), Orts- und Betriebskrankenkassen (1913-1945), Beamten- und Angestelltenbezïge (1925-1944) und Jahresverdienste von Landarbeitern (1912-1943).

Oberversicherungsamt Minden

DT, Best. M I OV

Generalakten (11 Bde, 1919-1940) mit Geschäftsberichten (bis 1935).

Oberversicherungsamt Sigmaringen

SIG, Best. Ho 262

Generalakten betr. Dienststellenverwaltung (24 Bde, 1933-1944) mit Geschäftsberichten und Erlassen des Reichsarbeitsministers betr. Krankenversicherung (33 Bde) und andere Versicherungszweige (15 Bde), Zuständigkeitsfragen (16 Bde, 1933-1942).

\subsubsection{Versorgungsverwaltung}

Von den Dienststellen im Geschäftsbereich des Reichsarbeitsministers, die für die Zahlung von Renten und HeilmaBnahmen für Kriegsopfer zuständig waren und zu denen 1942 im späteren Gebiet der Bundesrepublik und Berlins 7 Hauptversorgungsämter mit 46 Versorgungsämtern gehörten, scheint so gut wie keine geschlossene Überlieferung in die Archive gelangt zu sein (zum Reichsversorgungsgericht vgl. Abschnitt 3.5.2.2.3, zu den Fürsorge- und Versorgungsdienststellen der Wehrmacht 8.4.1.4, der SS 2.2.4.1). So sind z. B. von den sechs Versorgungsämtern der nördlichen Rheinprovinz aus der Zeit von 1933 bis 1945 nur wenige Bände von Essen und Köln vorhanden (D - K), und von den fünf westfälischen gibt es gar keine Bestände. Es ist allerdings damit zu rechnen, daß Einzelfallakten in erheblichem Umfang nach 1945 von den Länderbehörden fortgeführt wurden und in in deren Schriftgut enthalten sind. Eine Parallelüberlieferung liegt von der Hauptfürsorgestelle Detmold vor (DT, Best. L 80 I CHF, ca. 100 Bde, 1932-1945) u. a. mit Unterlagen über Tagungen der Leiter der Hauptfürsorgestellen (1936-1945), Arbeits- und Berufsfürsorge, vor allem Unterbringung im öffentlichen Dienst, Maßnahmen für Hirnverletzte und Blinde, Verwaltung von Darlehen und Spenden.

\subsection{Freiwilliger und Reichsarbeitsdienst}

Der seit 1931 vom Reich finanzierte Freiwillige Arbeitsdienst (FAD), der von Verbänden verschiedener Richtung getragen wurde, unter denen jedoch der "Stahlhelm" und der nationalsozialistische Reichsverband Deutscher Arbeitsdienstvereine e. V. nach der Zahl 
der Arbeitsdienstwilligen an der Spitze standen, kam ab 1933 unter die Kontrolle der NSDAP und wurde mit Einführung der Arbeitsdienstpflicht 1935 zum Reichsarbeitsdienst (RAD), der zugleich staatliche Einrichtung und Gliederung der NSDAP war. Die Überlieferung beider Organisationen ist äußerst spärlich. Relativ am besten ist der Einsatz des FAD und des Frauenarbeitsdienstes bis 1934 bzw. 1936 dokumentiert, weil darüber geführte Akten während des Krieges über die Arbeitsämter, die ihn finanziert hatten, den Staatsarchiven angeboten und, soweit als archivwürdig angesehen, von ihnen übernommen wurden; sie sind in den meisten Fällen mit Schriftgut von Arbeitsämtern vereinigt oder bilden sogar deren einzige Überlieferung aus der Zeit vor 1945.

Lit: H. CROON: Aktenhaltung und Archivgutpflege im Reichsarbeitsdienst. 1950. - W. BENZ: Vom freiwilligen Arbeitsdienst zum Arbeitsdienstpfichtgesetz 1968. - H. KÖHLER: Arbeitsdienst in Deutschland. 1967.

7.2.1 Reichsleitung

$\mathrm{BA}-\mathrm{ZNS}$

Der Bestand ( $R$ 77) enthält nur wenige Akten der Behörde selbst, an deren Spitze der Reichsarbeitsführer (bis 1934 Reichskommissar für den FAD, bis 1943 Staatssekretär im Reichsinnenministerium, dann in der Stellung eines Reichsministers) und Reichsleiter der NSDAP Konstantin Hierl stand, und einiges aus Empfangerüberlieferung damit vereinigtes Schriftgut.

Dazu gehören wenige Unterlagen über die Organisation (ergänzt durch eine Ausarbeitung des Leiters der Archiv- und Schriftgutverwaltung des RAD H. Croon von 1956), Protokolle der Tagungen der Gauarbeitsführer 1934 und 1935 (ein Protokoll einer Dienstbesprechung des Erziehungs- und Ausbildungsamtes 1944 in Best. RD 20), Akten über Erfassung, Musterung und Einsatz der weiblichen Jugend (10 Bde, 1939-1944). Amtsbefehle des Zeugamtes (1938-1945), ein Lehrgangsprogramm der Reichsschule Potsdam (1941), Handakten des Generalarbeitsführers v. Wenckstern aus dem Einsatz des RAD als Bautruppen der Wehrmacht im Osten (1939), der Tätigkeitsbericht des beim Westwallbau eingesetzten Arbeitsgaus $W$ für 1938/39, Gaubefehle und Feldpostrundbriefe aller Arbeitsgaue (20 Bde, 1933-1945) und Mitteilungen über Personalveränderungen in chronologischen (22 Bde, 1935-1945) und nach Arbeitsgauen (12 Bde) gebildeten Serien mit ca. 190000 Namen. Ca. 12000 Personalakten und Stamm(Gebührnis)akten von Führern, Angestellten und Arbeitern stammen von verschiedenen Arbeitsgauen, vor allem VIII, Ostmark (Frankfurt/Oder), XVIII, XIX, XXI und XXXI (Personalunterlagen über 1887 Führer des Arbeitsgaus X, Niederschlesien, im Berlin Document Center).

Der Hauptteil der Sachakten des Bestandes besteht aus Aktenresten (ca. 170 Bde) einer gröBeren Anzahl von einzelnen RAD-Gruppen und -Abteilungen oder unbestimmbarer Provenienz. Überwiegend (ca. 90 Bde) betreffen sie Mobilisierungs- und andere Reichsverteidigungsangelegenheiten (ab 1937), vor allem Abwehr von Spionage und Sabotage, auch Luftschutz. Der Rest (dabei einzelne Einsatztagebücher) gibt u. a. Aufschluß über die personelle und materielle Ausstattung und militärischen Einsatz.

Nach Potsdam (ZStA, Best. 15.20) gelangten lediglich wenige Akten über Personalangelegenheiten (3 Bde, 1936-1941) und ein Bildbericht über den Aufbau des norwegischen Arbeitsdienstes (1940-1941).

Der Nachlaß Hierls bietet nur eine wenig bedeutende Ergänzungsüberlieferung (BA. NL 125, meist nach 1945) mit Tagebuchnotizen (1939, 1941), Briefen an seine Frau (1938-1940), einzelnen Reden und Drucksachen (1941-1945) und einem Manuskript 
"Idee und Gestaltung des Arbeitsdienstes". Dasselbe gilt für den NachlaB des Inspekteurs für Verwaltung und Wirtschaft und der bei der Wehrmacht eingesetzten Einheiten, des Obergeneralarbeitsführers Herbert Schmeidler (BA, NL 50), der sich nach 1945 um die Ansprüche der RAD-Führer nach G 131 bemüht hat; er enthält Reste von Korrespondenz (1941-1944) mit Generalen und über Organisationsfragen sowie Reden und Vorträge (1938-1945). Der 1933 mit der Organisation des Arbeitsdienstes befaßte spätere Stabsleiter des Amtes Rosenberg, Helmut Stellrecht, hat eine Aufzeichnung über die Entstehung des RAD hinterlassen (BA, Kl. Erw. 599, auch IfZ, ZS 1906), der Oberstarbeitsführer (zuletzt im Arbeitsgau VII in Kiel) Hans Oeser Erinnerungen (BA, KI. Erw. 726).

Umfangreich war die Publizistik des RAD (im BA, Best. RD 20, meist nur durch einzelne Hefte vertreten). Vorschriften und Erlasse wurden im „Verordnungsblatt für den Reichsarbeitsdienst“ (1933-1945, bis April 1936 VOBl. der Reichsleitung des Arbeitsdienstes) und in einzelnen Dienst- (Dv, Da) und Technischen Vorschriften bekannt gegeben, die Daten der höheren Führer in den Dienstalterslisten (1937, 1938). „Der Arbeitsmann. Zeitung für Führer und Gefolgschaft" wandte sich ab 1935 an alle RADAngehörigen, die Führer erhielten seit 1931 die Führerzeitung ,Deutscher Arbeitsdienst für Volk und Heimat", ab 1942 „Führen und Erziehen“", dazu „Unterrichtsbriefe“ (19401944), das Sanitätspersonal ab 1939 den „Gesundheitsdienst im RAD“. Als Reihen gab es „Volk an der Arbeit“", „Der nationale Aufbau“ (30 Hefte bis 1941) und „Fest und Feier" (13 Hefte, 1942-1943), auch ein „Handbuch der Arbeitstechnik“ (105 Hefte bis 1943), jährlich bis 1938 den Bericht über die Teilnahme am Reichsparteitag und das „Jahrbuch des RAD“ (1-7, 1936-1943), dazu ein "Lehr- und Lesebuch“, Liederbücher, Berichte über einzelne Einsätze und Werbeschriften. Auch Filme (im BA - Filmarchiv) warben: "Arbeitsdienst ist Ehrendienst" (undatiert), riefen zum freiwilligen Eintritt in den weiblichen Arbeitsdienst $(1934,1938)$ auf und zeigten Bilder aus RAD-Schulen und vom Einsatz für die Wehrmacht in besetzten Gebieten („RAD-Filmbericht“, 14 Folgen, 1942-1943).

Lit.: GUIDES to German records. vol. 3. - SCHRIFTEN ZUM STAATSAUFBAU 14. 1941, 17. 1938. K. HIERL: Im Dienst für Deutschland. 1954. - R. SCHWENK: Geistige und materielle Grundlagen der Entstehung des Führerkorps im Arbeitsdienst und seine Gleichschaltung und Neuformung nach 1933. 1967.

\subsubsection{Arbeitsgaue}

Aus den 18 im späteren Gebiet der Bundesrepublik und Berlin (von insgesamt 32 im Reichsgebiet von 1937 gebildeten) zuständigen Arbeitsgauen für den Arbeitsdienst der Männer, die aus jeweils 5-10 Gruppen zu je 6-9 Abteilungen bzw. Lagern bestanden, ist Schriftgut meist nur durch die Abliefenungen von Akten aus der Frühzeit vorhanden; es fehlt völlig für den Arbeitsgau W, Köln, und vom Arbeitsgau IX, Brandenburg, Berlin-Lankwitz. Folgende Bestände oder Ersatzüberlieferungen waren zu ermitteln:

\section{Arbeitsgau VI, Schleswig-Holstein, Kiel}

Nur über den FAD im Bezirk Heide in Holstein sind Akten vorhanden (SL, Abt. 581). Sie betreffen Allgemeines (14 Bde, 1931-1933, dabei Führerschulung, Werbung, Statistik, Werkhalbjahr für Abiturienten 1933) und Einzelprojekte vor allem zur Landgewinnung und bei Meliorationsarbeiten, auch durch „Stahlhelm", Verein für Arbeitsdienst „Westküste“, Deutschnationaler Handlungsgehilfenverband (30 Bde, 1932-1934), Näharbeiten des weiblichen Arbeitsdienstes. 


\section{Arbeitsgau XVI, Westfalen-Nord, Münster}

FAD-Akten sind aus folgenden Arbeitsamtsbezirken überliefert (MS):

Bestwig: Allgemeines (8 Bde), Einzelprojekte (ca. 130 Bde).

Lippstadt: Pressesachen (5 Bde, 1932-1933), Lager Geseke und Stift Cappel des

Frauenarbeitsdienstes (3 Bde, 1934-1936), Einzelprojekte (36 Bde, 1932-1933).

Stadthagen: Einzelprojekte (8 in 2 Bden, 1933-1934, SHG, Des. L 41).

\section{Arbeitsgau XVII, Niedersachsen-Mitte, Bremen}

Seine Geschichte wird durch eine Sammlung von Unterlagen des ehemaligen Arbeitsdienstführers Boy Jensen vor allem über die einzelnen Lager dokumentier (HB, Best. $7,166,65$ Bde).

\section{Arbeitsgau XVIII, Niedersachsen-Ost, Hannover}

Die FAD-Akten (H, Best. Hann. 274 und WF, Best. 8 R mit unverzeichnetem Teilbestand über einzelne FAD-Projekte in verschiedenen Bezirken 1932-1935) stammen aus den Arbeitsamtsbezirken

Bassum: Einzelprojekte, auch des „Stahlhelm“ und des Jungdeutschen Ordens (8 Bde, 1930-1938).

Celle: Einzelprojekte (12 Bde, 1932-1934), Kassenbuch des Lagers Garßen (19331934).

Helmstedt (WF, Best. 8 R): Einzelprojekte (12 Bde, 1933-1934).

Personalakten von Führern befinden sich im Bestand des BA (ZNS).

Aus der publizistischen Tätigkeit des Gaus ist ein undatierter Film „Spaten und Ähren“ (BA - Filmarchiv) und ein Bericht „Arbeitsgau 18 marschiert“ (1934, mit Bildern, Karten und graphischen Darstellungen, BA) überliefert.

\section{Arbeitsgau XIX, Niedersachsen-West, Oldenburg}

Während über den FAD-Einsatz in den Arbeitsamtsbezirken Emden und Leer nur wenige Akten (AUR) über Einzelprojekte (Abrechnung von Meliorationsarbeiten, 15 Bde, 1932 1933, bzw. 11 Bde, 1932-1933) vorliegen, ist er gut dokumentiert für den Bezirk

Nordhorn (OS, Rep. 450): Allgemeines (9 Bde, 1932-1936, u. a. Führerbesprechungen, Abrechnungen, Statistiken), Einsatz des Frauenarbeitsdienstes (11 Bde, $1932-$ 1936, Verfügungen, Näharbeiten, Siedlungshilfe in vier Lagern), 20 vom RAD fortgesetzte Einzelprojekte von FAD, „Stahlhelm“, Jungdeutschem Orden, Katholischem Jungmännerverband (34 Bde, 1932-1938), 16 Einzelprojekte der NSDAP (22 Bde, 19321935). Ergänzungsüberlieferung enthalten Akten der Verwaltung des Kreises Grafschaft Bentheim (10 Bde, 1933-1941, Rep. 450) und eine Materialsammlung des ehemaligen Arbeitsdienstführers und Ingenieurs Heinrich Winkler über die Arbeiten im Emsland 1931 bis 1933 (OS, Kl. Erw. 57), die durch Aufzeichnungen und Tabellen über den Arbeitsgau fortgesetzt wird (Kl. Erw. 70).

\section{Arbeitsgau XX, Westfalen-Süd, Dortmund}

Überlieferung über den FAD ist für folgende Arbeitsamtsbezirke vorhanden (MS):

Arnsberg: Verfügungen und Rundschreiben (18 Bde, 1931-1934), Angelegenheiten von Führern (3 Bde, 1933-1934), des Westfälischen Arbeitsdienstwerks (4 Bde, 1932 1934) und des Frauenarbeitsdienstes (2 Bde, 1934-1935), Einzelprojekte (ca. 120 Bde, 1932-1933).

Bochum: Allgemeines (5 Bde), Einzelprojekte (7 Bde). 
Hagen: Verfügungen des Reichskommissars für den Arbeitsdienst (3 Bde, $1932-$ 1933) und des Bezirkskommissars (4 Bde, 1933-1934). Frauenarbeitsdienst (17 Bde, 1933-1936), Führerschulung (2 Bde, 1932-1933), Sozialversicherung (5 Bd, 1933), Einzelprojekte, auch des „Stahlhelm“ und der NS-Frauenschaft (ca. 200 Bde, 1932-1934).

Hamm: Allgemeines (3 Bde, 1931-1934), Einzelprojekte (10 Bde, 1933).

Kamen: Verfügungen (7 Bde, 1931-1933), allgemeine Personalangelegenheiten (7 Bde, 1932-1934), Geländesport (1935), Freizeitgestaltung (1932-1933), Einzelprojekte (29 Bde, 1931-1933).

Lüdenscheid: Angelegenheiten der Führer (3 Bde, 1932-1933), des Haushalts- und Kassenwesens (29 Bde, 1932-1934).

Recklinghausen: Pressesachen (2 Bde, 1931-1934), Tagungen (1932-1933), Unterrichtspläne (1933), Einzelprojekte (5 Bde, 1932-1933).

Siegen: Angelegenheiten des Führerschulungslager Herdorf (Sieg) und einzelner Lager, auch des „Stahlhelm“, des Nationalen Arbeitsdienstes „Rote Erde“ und des Jungdeutschen Ordens (39 Bde, 1932-1935).

\section{Arbeitsgau XXI, Niederrhein, Düsseldorf}

Akten (D - K) betreffen den FAD-Einsatz in folgenden Arbeitsamtsbezirken:

Erkelenz: Allgemeines (30 Bde, 1932-1934, dabei Führerschulung, Besichtigung und ärztliche Betreuung der Lager, Lohnlisten), Einzelprojekte (19 Bde, 1932-1934).

Eschweiler: Allgemeines (1932-1933), Einzelprojekte (13 Bde, 1931-1933).

Moers: Allgemeines (8 Bde, 1932-1934), Einzelprojekte (52 Bde, 1931-1934).

Gaubefehle und Feldpostrundbriefe (2 Bde, 1934-1944), Mitteilungen über Personalveränderungen (3 Bde, 1939-1944) sowie Personalakten von Führern, Angestellten und Arbeitern befinden sich :m Bestand des BA (ZNS).

\section{Arbeitsgau XXI, Hessen-Nord, Kassel}

Die Überlieferung (MR, Best. 604, 1, 2, 3 und 4) stammt aus den Bezirken der Arbeitsämter

Fulda: Allgemeines (4 Bde, 1933-1934), Frauenarbeitsdienst (3 Bde, 1933-1936), Einzelprojekte (3 Bde, 1933-1934).

Korbach: Allgemeines (6 Bde, 1933-1934, dabei Heimatwerk für Hessen-Nassau, Führerschulung), Frauenarbeitsdienst (1933-1936), Einzelprojekte (2 Bde, 1933-1934).

Marburg: Allgemeines (5 Bde, 1933-1934, dabei Schulungskurse 1933-1936, schwarze Listen 1932-1933), Frauenarbeitsdienst (1933-1935), Einzelprojekte (2 Bde, 19331934).

Treysa: Einzelprojekte (2 Bde).

\section{Arbeitsgau XXIV, Mittelrhein, Koblenz}

AuBer einigen Unterrichtsplänen (1934-1935), Gaubefehlen und Feldpostrundbriefen im Mischbestand (BA-ZNS) gibt es nur noch eine Gau-Chronik von etwa 1935 (KO).

Lit.: H. KNEBEL: Zur Geschichte des Arbeitsdienstes 1933-1945. 1987.

\section{Arbeitsgau XXV, Hessen-Süd, Wiesbaden}

Während von der Führung des Gaus nur eine Aufzeichnung des Richterlichen RAD. Führers über Strafrecht im Kriegseinsatz (1940-1942) und Befehle (BA-ZNS), ferner Handakten des Oberstarbeitsfuhrers Gustav Zahnow (1933-1944, mit Befehlen von 1939) und Personalakten von 25, vor allem aus dem Gau XXXI, dorthin versetzten 
Führem vorhanden sind (WI, in Abt. 483), ist von der unterstellten Gruppe 257, Wiesbaden, etwas mehr überliefert (ebda, 45 Bde, 1938-1942, vor allem betr. Personalangelegenheiten einschlieBlich politischer Erziehung und Disziplinarsachen, mit Gruppenund Stabsbefehlen 1939-1942). Die Akten über 87 Einzelprojekte des FAD (1932-1933) u. a. in Bad Homburg. Eppstein, Oberursel und Wiesbaden wurden als Schriftgut des Landesarbeitsamtes Hessen in einem Bestand vereinigt (WI, Abt. 479), der auch Unterlagen (5 Bde, 1933) über Fortbildungslehrgänge des Notwerks der deutschen Jugend in den Arbeitsamtsbezirken Niederlahnstein, Mainz und Limburg enthält.

\section{Arbeitsgau XXVI, Württemberg, Stuttgart}

Einige wenige Akten (LB, Best. K 176, 9 Bde, 1933-1944) betreffen Aus- und Weiterbildung, einzelne Lager, Personalangelegenheiten, die Stiftung „Arbeitsdank“ und Darlehen an Führer. Zu einem gröBeren Bestand (Best. 310/1 II, ca. 600 Bde, 1932-1940) wurden Akten über FAD und Frauenarbeitsdienst im allgemeinen (mit Runderlassen, Unterlagen über Versicherung, Abrechnung der Mittel nach Lagern, Projekten und Bezirken) und über den Einsatz in folgenden Arbeitsamtsbezirken vereinigt:

Stuttgart (1933-1934, dabei Führertagungen), Esslingen (1932), Ulm (1932-1935), Ravensburg (1931-1935, mit ErlaBsammlung), Ludwigsburg (1931-1935), Heilbronn (1932-1934, mit Runderlassen), Balingen (1932-1934), Schwäbisch Hall (1932-1934), Bad Mergentheim (1931-1935), Aalen (1931-1934), Schwäbisch Gmünd (1932), Nagold (1932-1934), Rottweil (1931-1933), Göppingen (1932-1933), Biberach an der RiB (1933-1935). Außerdem enthält der Bestand Monatsberichte des RAD für die weibliche Jugend aus den Lagern Böckingen, Schmidhausen und Güglingen (1939-1941).

Anderweitig überliefert sind die Akten aus dem Bezirk Sigmaringen (SIG, Best. Ho 370) über Allgemeines und Einzelprojekte (41 Bde, 1931-1937, auch über „Stahlhelm-“Arbeitsdienst und einzelne Lager, Übernahme durch den RAD).

\section{Arbeitsgau XXVI, Baden, Karlsruhe}

Einzelne Befehle und Rundschreiben (1937-1939 IfZ, Best. Fa 288), Akten (KA, Best. 460) über den Einsatz des FAD in folgenden Arbeitsamtsbezirken:

Karlsruhe: Allgemeines, (dabei Einführung der Arbeitsdienstpflicht) und Einzelprojekte (insges. ca. 500 Bde, 1927-1955).

Mannheim: Einzelprojekte (6 Bde, 1931-1937).

Offenburg: Allgemeines (dabei Katholisches Arbeitswerk und Heimatwerk Baden), Frauenarbeitsdienst und Einzelprojekte (28 Bde, 1933-1937).

Pforzheim: Allgemeines (dabei NS-Arbeitsgauverein 1933, Schulungslager) und Einzelprojekte (92 Bde, 1932-1933).

Rastatt: Einzelprojekte (35 Bde, 1931-1936).

Personalakten von Führern befinden sich im Bestand des BA.

\section{Arbeitsgau XXVII, Franken, Würzburg}

Akten aus diesem Gau und vor allem seiner Gruppe 280, Kulmbach, und ihrer Einheiten bilden den Hauptteil des Mischbestandes (im BA-ZNS); dazu gehören auch Befehlsund Erlaßsammlungen (Dienstanweisungen der Gruppe 280, 1939, auch BAM). Ferner sind Akten (N, Rep. 251 III) über den Einsatz des FAD im allgemeinen (3 Bde) und bei Einzelprojekten im Arbeitsamtsbezirk Nürnberg, u. a. in Nürnberg, Schwabach und Erlangen (37 Bde) sowie Personalangaben über alle Führer im Gau Bayern-West (1933, ebda, Rep. 503 RAD) überliefert. 
Lit: F. SCHINNERER: Unser Arbeitsgau 28 (Franken). 1935.

Arbeitsgau XXIX, Bayern-Ostmark, Regensburg

Die Akten wurden von folgenden Arbeitsämtern abgeliefert:

Cham: Einzelprojekte (AM, 13 Bde, 1933-1934).

Hof: Allgemeines über wertschaffende Arbeitslosenfürsorge (BAM, 9 Bde, 1933 1934) und Einzelprojekte, auch der NSDAP und des „Stahlhelm“ (29 Bde, 1932-1934), Einsatz des Deutschen Frauenarbeitsdienstes (Lager Reitzenstein, 11 Bde, 1933-1936).

Marktredwitz: Allgemeines und Lager Sophienreut (BAM, 4 Bde, 1933-1937), Einzelprojekte (15 Bde, 1933-1934).

Arbeitsgau XXX, Bayern-Hochland, München

Die Überlieferung ist auf einige Befehle und Rundschreiben (1936-1943) beschränkt (IfZ, Best. Fa 288).

\section{Arbeitsgau XXXII, Saar-Pfalz, Münster am Stein}

Akten (SP, Best. O 1, 2 und 3) betreffen den FAD-Einsatz in den Arbeitsamtsbezirken

Kaiserslautern: Einzelprojekte, insbesondere Bau von Sportanlagen (30 Bde, 1932 1933), Frauenarbeitsdienst (5 Bde, 1933-1937).

Landau: Allgemeines (5 Bde, 1933), Schulung (1932-1938), Frauenarbeitsdienst (2 Bde, 1933-1937).

Pirmasens: Allgemeines (1931-1933), Einzelprojekte (9 Bde, 1931-1933), Frauenarbeitsdienst (1933-1936).

Gaubefehle (1944) befinden sich im Mischbestand (BA-ZNS).

\subsubsection{Bezirke des Reichsarbeitsdienstes für die weibliche Jugend}

Die Überlieferung für die 10 Bezirke (bis 1936 des Deutschen Frauenarbeitsdienstes) im Gebiet der späteren Bundesrepublik aus der Zeit nach Einführung der Dienstpflicht 1939 ist noch dürftiger und beschränkt sich auf Stamm(Gebührnis)Karten von Führerinnen aus dem Bezirk VIII, Hannover (ca. 800, BA-ZNS), auf Mitteilungen über Personalveränderungen und Rundverfügungen (1942) aus dem Bezirk X, Koblenz (ebda), Rundschreiben (1939) des Bezirks XI, Wiesbaden (WI, in Abt. 483) und aus dem fränkischen Arbeitsgau XXVIII (BA-ZNS, 5 Bde, 1940-1945), Personalveränderungsmitteilungen (1944) aus Bezirk XIX, Würzburg (ebda).

Für sieben westdeutsche Bezirke sind jedoch Erinnerungsbücher erschienen.

Lit: D. G. MORGAN: Weiblicher Arteitsdienst in Deutschland. 1978. - L. KLEIBER: „Wo ibr seid, soll die Sonne scheinen!“ 1981. - S. BAJOHR: Weiblicher Arbeitsdienst im .Dritten Reich“. 1980. - E. ECKERT: Arbeitsdienst für die weibliche Jugend - Antworten nach 40 Jahren. 1974. - H. BERENDT-HAAS: Wir erinnern uns. (um 1980). - L. BENEDIX: Wur Arbeitsmaiden in Hessen. (um 1942). - A. HAMMER: Chronik des Bezirks XII, Württemberg, Reichsarbeitsdienst w. J. 1975. - ARBEITSMAID in der Nordmark. 1940. - L GOETZE, 1. MÜTZE: Damals in Weser-Ems. 1978. - M. WEIB: Arbeitsmaiden am Obenthein. um 1943. 
